EPJ Web of Conferences 37, 09020 (2012)

DOI: $10.1051 /$ epjconf/20123709020

(C) Owned by the authors, published by EDP Sciences, 2012

\title{
Recent results from SND experiment at VEPP-2000 collider
}

M.N. Achasov ${ }^{1,2}$, V.M. Aulchenko ${ }^{1}$, A.Yu. Barnyakov ${ }^{1}$, K.I. Beloborodov ${ }^{1,2}$, A.V. Berdyugin ${ }^{1,2}$, D.E. Berkaev ${ }^{1,2}$, A.G. Bogdanchikov ${ }^{1}$, A.A. Borisov ${ }^{1}$, A.A. Botov ${ }^{1}$, D.A. Bukin ${ }^{1}$, T.V. Dimova ${ }^{1}$, V.P. Druzhinin ${ }^{1,2}$, V.B. Golubev ${ }^{1,2}$, K.I. Grevtsov ${ }^{1,2}$, L.V. Kardapoltsev ${ }^{1,2, a}$, A.S. Kasaev ${ }^{1}$, A.G. Kharlamov ${ }^{1,2}$, A.N. Kirpotin ${ }^{1}$, I.A. Koop ${ }^{1,2}$, A.A. Korol ${ }^{1,2}$, S.V. Koshuba ${ }^{1}$, D.P. Kovrizhin ${ }^{1}$, E.A. Kravchenko, ${ }^{1,2}$, K.A. Martin ${ }^{1,3}$, A.E. Obrazovsky ${ }^{1}$, A.P. Onuchin ${ }^{1,3}$, A.V. Otboev ${ }^{1}$, E.V. Pakhtusova ${ }^{1}$, Yu.A. Rogovsky ${ }^{1,2}$, S.I. Serednyakov ${ }^{1,2}$, Yu.M. Shatunov ${ }^{1}$, D.A. Shtol ${ }^{1}$, D.B. Shwartz ${ }^{1,2}$, Z.K. Silagadze ${ }^{1,2}$, K.Yu. Skovpen ${ }^{1}$, A.N. Skrinsky ${ }^{1}$, I.K. Surin ${ }^{1,3}$, Yu.A. Tikhonov ${ }^{1,2}$, Yu.V. Usov ${ }^{1}$, and A.V. Vasiljev ${ }^{1,2}$

1 Institute of Nuclear Physics, Novosibirsk, 630090, Russia

2 Novosibirsk State University, Novosibirsk, 630090, Russia

3 Novosibirsk State Technical University, Novosibirsk, 630092, Russia

\begin{abstract}
Preliminary results of the SND experiment at the VEPP-2000 $e^{+} e^{-}$collider are presented. Data corresponding to about $40 \mathrm{pb}^{-1}$ of integrated luminosity were collected in the $e^{+} e^{-}$c.m. energy range from 1.05 to $2.00 \mathrm{GeV}$. The cross sections for $e^{+} e^{-} \rightarrow \omega \pi^{0}, \pi^{+} \pi^{-} 4 \pi^{0}, p \bar{p}, n \bar{n}$ have been measured.
\end{abstract}

\section{Introduction}

The $e^{+} e^{-}$collider VEPP-2000 [1] is aimed to make high precision measurements of hadronic cross sections in the energy range up to $2 \mathrm{GeV}$. The precise measurements of these cross sections is needed to improve the accuracy of the calculation of the hadronic contribution to the muon anomalous magnetic moment [2]. Detailed study of the energy region between 1 and $2 \mathrm{GeV}$, where $\rho, \omega$, and $\phi$ excitations are located, will provide information about their properties as well as clarify the problem of the possible existence of light vector hybrids below $2 \mathrm{GeV}$. Measurement of the nucleon-antinucleon production cross sections and other hadronic cross sections near $N \bar{N}$ threshold is needed for better understanding $N \bar{N}$ forces and resonances. More details about the VEPP-2000 physical program can be found in Ref. [4]. Two detectors, SND [3] and CMD-3 [4], collect data at VEPP-2000 since 2010.

\section{Experiment}

SND [3] is a general purpose non-magnetic detector. Its main part is a spherical three-layer NaI(Tl) calorimeter with 560 individual crystals per layer and $90 \%$ solid angle coverage. The energy resolution for photons is $\sigma_{E} / E=4.2 \% / \sqrt[4]{E(G e V)}$, the angular resolution $\simeq 1.5^{\circ}$. There is a tracking system around the collider beam pipe, based on nine-layer drift chamber and one-layer proportional chamber with cathode strip readout. Outside of the calorimeter a muon detector, consisting of proportional tubes and scintillation counters is placed. An aerogel Cherenkov counter located between the drift chamber and the calorimeter is used for particle identification.

The experiments at VEPP-2000 started in 2010. During 2010- 2012 the energy range $2 E=1.05-$ $2.00 \mathrm{GeV}$ was scanned with the step of $20-25 \mathrm{MeV}$. The achieved instant luminosity was $0.8 \times 10^{31}$ $\mathrm{cm}^{-2} \mathrm{~s}^{-1}$ near $1 \mathrm{GeV}$ and $2 \times 10^{31} \mathrm{~cm}^{-2} \mathrm{~s}^{-1}$ at $2 \mathrm{GeV}$. Total integrated luminosity collected by SND is about $40 \mathrm{pb}^{-1}$.

\footnotetext{
a e-mail: 1.kardapoltsev@gmail.com
}

This is an Open Access article distributed under the terms of the Creative Commons Attribution License 2.0, which permits unrestricted use, distribution, and reproduction in any medium, provided the original work is properly cited. 


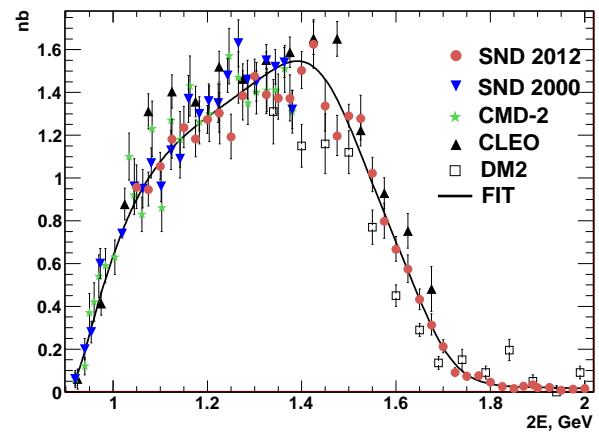

Fig. 1. The cross section for $e^{+} e^{-} \rightarrow \omega \pi^{0} \rightarrow \pi^{0} \pi^{0} \gamma$ measured in this work (SND 2012) and other experiments: SND 2000 [6], CMD-2 [7], DM2 [8], CLEO [9]. The curve is the result of the fit to SND 2012 and SND 2000 data.

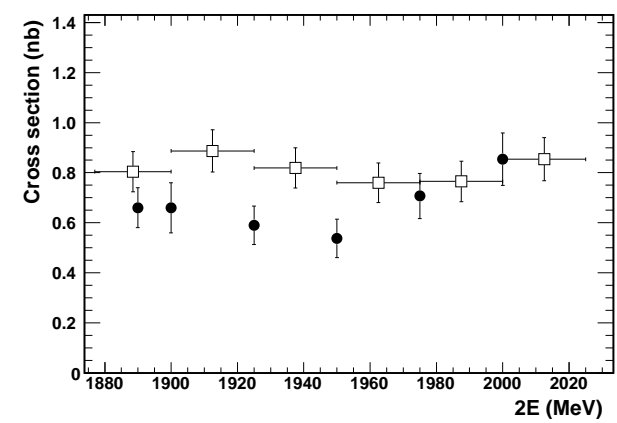

Fig. 2. The $e^{+} e^{-} \rightarrow p \bar{p}$ cross section measured by SND (squares) and BABAR [10] (circles).

\section{$3 e^{+} e^{-} \rightarrow \omega \pi^{0} \rightarrow \pi^{0} \pi^{0} \gamma$}

The process $e^{+} e^{-} \rightarrow \omega \pi^{0} \rightarrow \pi^{0} \pi^{0} \gamma$ is studied using $27 \mathrm{pb}^{-1}$ data collected in 2010 and 2011 . The previous SND measurement [5] was based on data of 2010 corresponding to $5 \mathrm{pb}^{-1}$.

The cross section for $e^{+} e^{-} \rightarrow \omega \pi^{0} \rightarrow \pi^{0} \pi^{0} \gamma$ measured by SND is shown in Fig. 1 in comparison with results of other experiments. Our data are in good agreement with the measurements [6,7] performed by SND and CMD-2 at the VEPP-2M collider at energies below $1.4 \mathrm{GeV}$, but significantly (by 20-30\%) exceed DM2 [8] data, only previous measurement of the $e^{+} e^{-} \rightarrow \omega \pi^{0}$ cross section at energies above $1.4 \mathrm{GeV}$.

We also present comparison our cross-section data with the cross section calculated under CVC hypothesis from the spectral function of the $\tau \rightarrow \omega \pi v_{\tau}$ decay measured in the CLEO experiment [9]. A sizable difference is observed between $e^{+} e^{-}$and $\tau$ data.

\section{$4 e^{+} e^{-} \rightarrow p \bar{p}, n \bar{n}$}

The $e^{+} e^{-} \rightarrow p \bar{p}$ process is studied using about $3 \mathrm{pb}^{-1}$ collected in 2011 above $p \bar{p}$ threshold. With the selection criteria used the detection efficiency is about $40 \%$. The background estimated using data below the threshold is about 6\%. The measured cross section is shown in Fig. 2 in comparison with BABAR data [10].

The $e^{+} e^{-} \rightarrow n \bar{n}$ process is studied with a part $\left(2.5 \mathrm{pb}^{-1}\right)$ of 2011 data. The detection efficiency for $n \bar{n}$ events is about $25 \%$. The cross section obtained after background subtraction shown in Fig.3 is fitted with a step function. The fit gives $\sigma=0.1 \pm 0.2 \mathrm{nb}$ below and $\sigma=0.8 \pm 0.2 \mathrm{nb}$ above the $n \bar{n}$ threshold. Our result agrees with FENICE measurement [11]. The quoted errors are statistical. Systematic uncertainties are to be analyzed but as it is anticipated they could be $\sim 40 \%$.

\section{$5 e^{+} e^{-} \rightarrow \pi^{+} \pi^{-} 4 \pi^{0}$}

The interest in $e^{+} e^{-}$annihilation into 6 pions is motivated by observation of an interference structure in the cross sections for $e^{+} e^{-} \rightarrow 3\left(\pi^{+} \pi^{-}\right)$and $e^{+} e^{-} \rightarrow 2\left(\pi^{+} \pi^{-} \pi^{0}\right)$ near nucleon-antinucleon threshold. This structure was discovered by DM2 [12] and then confirmed by BABAR [13]. The third six-pion process, $e^{+} e^{-} \rightarrow \pi^{+} \pi^{-} 4 \pi^{0}$, was not studied experimentally.

The visible cross section for $e^{+} e^{-} \rightarrow \pi^{+} \pi^{-} 4 \pi^{0}$ measured by SND is shown on Fig. 4. A part of $e^{+} e^{-} \rightarrow \pi^{+} \pi^{-} 4 \pi^{0}$ events originating from the process $e^{+} e^{-} \rightarrow \pi^{+} \pi^{-} \pi^{0} \eta$ with decay $\eta \rightarrow 3 \pi^{0}$ 


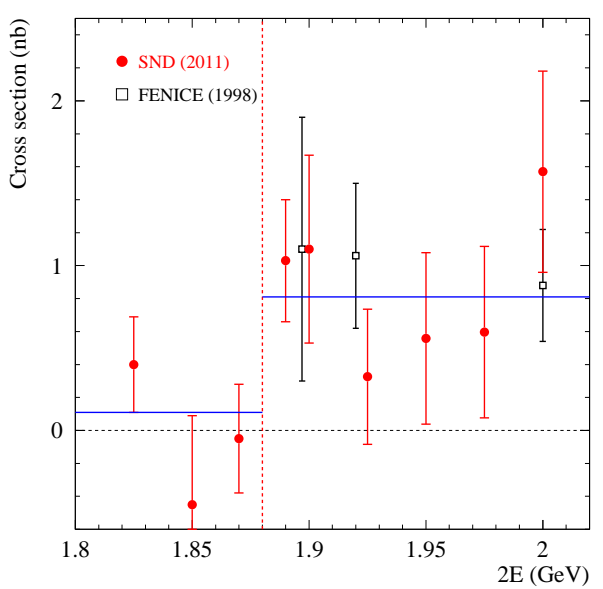

Fig. 3. The $e^{+} e^{-} \rightarrow n \bar{n}$ cross section measured by SND and FENICE [11] (squares).

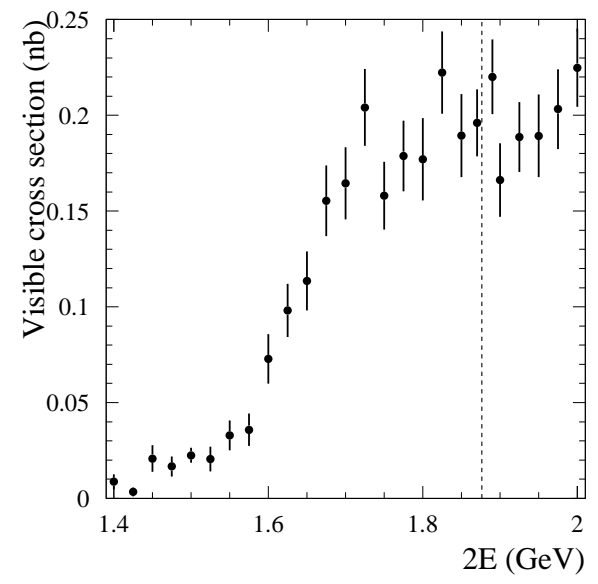

Fig. 4. The visible cross section for selected events of the $e^{+} e^{-} \rightarrow \pi^{+} \pi^{-} 4 \pi^{0}$ process. The vertical dashed line indicates the $p \bar{p}$ threshold.

was removed by the cut $0.51<M_{3 \pi^{0}}<0.59 \mathrm{GeV} / \mathrm{c}^{2}$. At the present level of statistical accuracy the interference structure near $N \bar{N}$ threshold is not seen in the $e^{+} e^{-} \rightarrow \pi^{+} \pi^{-} 4 \pi^{0}$ cross section.

\section{Conclusions}

Data with integrated luminosity of about $40 \mathrm{pb}^{-1}$ have been collected by SND detector at VEPP-2000 $e^{+} e^{-}$collider in the energy range $2 E=1.05-2.00 \mathrm{GeV}$. The preliminary results on the cross sections for $e^{+} e^{-} \rightarrow \omega \pi^{0}, \pi^{+} \pi^{-} 4 \pi^{0}, p \bar{p}, n \bar{n}$ have been obtained.

This work is supported by the Ministry of Education and Science of the Russian Federation, the Russian Federation Presidential Grant for Scientific Schools NSh-6943.2010.2, RFBR (grants 11-0200276-a, 11-02-09588-mob-z, 12-02-01250-a, 12-02-00065-a) and the Russian Federation Presidential Grant for Young Scientists MK-4345.2012.2.

\section{References}

1. Yu. M. Shatunov et al., in Proceedings of the 7th European Particle Accelerator Conference, Vienna, 2000, p.439.

2. T. Kinoshita, B. Nizic and Y. Okamoto, Phys. Rev. D 31 (1985) 2108.

3. M. N. Achasov et al., Nucl.Instrum.Meth. A 598, 31 (2009); V. M. Aulchenko et al., Nucl.Instrum.Meth. A 598, 102 (2009); A. Yu. Barnyakov et al., Nucl.Instrum.Meth. A 598, 163 (2009); V. M. Aulchenko et al., Nucl.Instrum.Meth. A 598, 340 (2009).

4. B. Khazin, Nucl. Phys. Proc. Suppl. 181-182, 376 (2008).

5. M. N. Achasov et al., JETP Lett. 94, 2 (2012).

6. M. N. Achasov, K. I. Beloborodov, A. V. Berdyugin et al., Phys. Lett. B 486, 29 (2000).

7. R. R. Akhmetshin, V. M. Aulchenko, V. Sh. Banzarov et al., Phys. Lett. B 562, 173 (2003).

8. D. Bisello et al., Nucl. Phys. Proc. Suppl. 21, 111 (1991).

9. K. W. Edwards et al., Phys. Rev. D 61, 072003 (2000).

10. B. Aubert et al. Phys. Rev. D 71, 012005 (2006).

11. A. Antonelli et al. (FENICE Collaboration), Phys. Lett. B 313, 283 (1993); Nucl. Phys. B 517, 3 (1998).

12. R. Baldini et al., reported at the "Fenice" Workshop, Frascati (1988).

13. B. Aubert et al. (BABAR Collaboration), Phys. Rev. D 73, 052003 (2006). 